\title{
La Révolution française à l'heure du global turn
}

Paul Cheney, Alan Forrest, Lynn Hunt, Matthias Middell et Karine Rance

Traducteur : Karine Rance

(2) OpenEdition

Journals

Édition électronique

URL : https://journals.openedition.org/ahrf/12988

DOI : $10.4000 /$ ahrf. 12988

ISSN : 1952-403X

Éditeur :

Armand Colin, Société des études robespierristes

Édition imprimée

Date de publication : 1 décembre 2013

Pagination : 157-185

ISBN : 978290832789

ISSN : 0003-4436

Référence électronique

Paul Cheney, Alan Forrest, Lynn Hunt, Matthias Middell et Karine Rance, « La Révolution française à

I'heure du global turn », Annales historiques de la Révolution française [En ligne], 374 I octobre-décembre 2013, mis en ligne le 01 décembre 2016, consulté le 01 juillet 2021. URL : http://

journals.openedition.org/ahrf/12988; DOI : https://doi.org/10.4000/ahrf.12988 
REGARDS CROISÉS

\title{
LA RÉVOLUTION FRANCGAISE À L'HEURE DU GLOBAL TURN
}

\author{
Paul CHENEY \\ Alan FORREST \\ Lynn HUNT \\ Matthias MIDDELL \\ Karine RANCE
}

Donner la parole à quatre historiens étrangers, Lynn Hunt (UCLA), Paul Cheney (Université de Chicago), Alan Forrest (Université de York), et Matthias Middell (Université de Leipzig) pour débattre des potentialités de l'histoire globale pour l'étude de la Révolution française traduit une volonté de décentrement autant qu'une situation éditoriale : Paul Cheney a publié en 2010 Revolutionary Commerce. Globalization and the French Monarchy (Harvard University Press, compte rendu dans le n³69, 2012 des $A H R F)$; Matthias Middell vient de publier avec Luis Roura Transnational Challenges to National History Writing (Palgrave Macmillan, 2013) et prépare avec Alan Forrest un ouvrage sur l'histoire globale de la Révolution française (Routledge, 2014) ; Lynn Hunt a publié également cette année avec Suzanne Desan et William Max Nelson, The French Revolution in Global Perspective (Cornell Univ. Press, 2013).

Dans la même veine se trouvent les travaux de David Armitage sur une histoire globale de la Révolution ${ }^{1}$, ceux de David Geggus qui situe la révolution haïtienne au cœur du processus révolutionnaire, et bien d'autres qui, au-delà de nos frontières, s'enthousiasment pour une perspective (globale, connectée, entangled, croisée, transnationale, etc.) qui est loin de faire l'unanimité en France.

(1) David Armitage, Sanjay Subrahmanyam (dir.), The Age of Revolutions in Global Context, c. 1760-1840, Palgrave Macmillan, 2010. Compte rendu de Annie Jourdan dans le numéro 373 (2013-3) des $A H R F$.

ANNALES HISTORIQUES DE LA RÉVOLUTION FRANÇAISE - 2013 - No 4 [157-185] 
Pourquoi un tel engouement ailleurs ? Est-ce juste un effet de mode, ou est-ce que s'affranchir du cadre national permet réellement de poser des questions pertinentes ? Peut-on travailler sur la Révolution française et échapper au tropisme national, ou même européen ? La question est donc de savoir ce que le global turn peut apporter de nouveau sur la Révolution française.

\section{Karine Rance}

Le débat sur la Révolution atlantique, ouvert dans le contexte de la guerre froide par Robert Palmer et Jacques Godechot, a été relancé notamment en France à nouveaux frais dans les années $2000^{2}$. À l'étranger aussi l'histoire atlantique retrouvait un nouveau souffle. Y a-t-il une filiation entre l'histoire atlantique de Robert Palmer et Jacques Godechot et l'histoire connectée ou globale actuelle?

\section{Paul Cheney}

L'histoire extra-hexagonale de la Révolution française a précédé les innovations historiographiques de l'histoire « connectée » ou « globale ». Robert R. Palmer a écrit la première histoire globale de la Révolution française, l'Age des Révolutions démocratiques. Il étend son étude à des espaces très vastes allant de l'Afrique du Sud néerlandaise et de l'Inde Britannique à Java. Même si pour lui la Révolution française a suscité une « agitation universelle », son objet principal est la " civilisation occidentale » qu'il concevait comme un phénomène atlantique ${ }^{3}$. Étant donné que c'est Palmer et Godechot qui ont initialement défini l'histoire atlantique et déterminé ses bases ${ }^{4}$, on peut difficilement reprocher à l'Age des Révolutions démocratiques d'être une histoire globale ratée.

Palmer a présenté la Révolution démocratique - et la Révolution française, qui en a été la manifestation principale et celle qui a provoqué la plus grande rupture - comme une histoire principalement politique et intellectuelle. Franco Venturi ${ }^{5}$ a une approche similaire, sur le plan méthodologique et géographique. L'ensemble de son œuvre est certainement plus

(2) Claude MAZAURIC, « Aperçu des tendances et des enjeux historiographiques : le nécessaire débat », Cahiers d'histoire. Revue d'histoire critique. Numéro spécial coordonné par Michel BIARD, Des révoltes de l'Europe à l'Amérique au temps de la Révolution française 1773 à 1802, p. 19-25. Voir aussi les Annales HSS 2012/2 sur l'histoire atlantique.

(3) Robert R. PALmER, The Age of Democratic Revolution: a Political History of Europe and America, 1760-1800, Princeton, Princeton University Press, 1959, vol. 1, p. 5-7.

(4) Bernard BAIlyn, Atlantic History, Concepts and Contours, Cambridge, Mass., Harvard University Press, 2005.

(5) Franco VENTURI, Settecento Riformatore, 1969. 
kaléidoscopique, et peut-être intellectuellement plus subtile que celle de Palmer car il n'est pas orienté (ou alourdi ?) par un grand récit. Et parce qu'il porte attention au jeu entre le centre et la périphérie, ainsi qu'aux petits états, Venturi se rapproche davantage d'une sorte d'histoire connectée, qu'on associe généralement à Sanjay Subrahmanyam ${ }^{6}$. Mais alors que ce dernier examine le jeu des influences mutuelles et du métissage culturel à travers l'espace impérial, Venturi concentre son attention sur l'interaction entre les petits et les grands États européens, et sur le rôle des intellectuels cosmopolites - parfois des élites administratives - dans la formation d' un mouvement de réforme pan-européen antérieur à la Révolution française. Il y a donc des antécédents à l'histoire connectée ou globale des Lumières et de la Révolution française que nous discutons ici, et Venturi offre peut-être aux historiens un modèle plus viable que Palmer.

\section{Lynn Hunt}

Histoire connectée, transnationale, globale, impériale, atlantique ou world history ont fait l'objet d'un nombre croissant d'articles, de conférences et de livres aussi bien en français qu'en anglais. La Révolution française occupe souvent une place particulière dans ces discussions. Contrairement aux années 1950, quand la Révolution française était au cœur des débats sur l'histoire atlantique (grâce aux écrits de Robert R. Palmer et de Jacques Godechot), les discussions récentes ne sont pas issues des débats portant sur l'interprétation de la Révolution française. Et pourtant, même si les débats actuels ont d'autres origines - questionnement post-colonial sur l'immigration, les diasporas et l'identité, redécouverte de l'importance des conflits entre empires, curiosité ou défiance à l'égard de la globalisation, effort pour incorporer l'histoire de l'esclavage dans des récits sur l'émergence des États-nations modernes - ces débats sont loin d'être sans rapport avec la Révolution française. Personne ne nie, par exemple, que la lutte coloniale avec la Grande Bretagne et les effets des emprunts faits pendant la guerre d'Indépendance américaine entre 1778 et 1783 sont des causes majeures de la révolution de 1789. Pourtant les historiens de la Révolution ont souvent été lents à s'emparer de questions générales dont les spécialistes en histoire américaine ou britannique s'étaient déjà emparés avec un certain enthousiasme.

(6) Sanjay Subrahmanyam, Explorations in Connected History. Oxford, Oxford University 


\begin{abstract}
Alan Forrest
Il n'y a pas de continuité directe, à mon avis, entre la réflexion des années 1950-1960 sur les fondements de la démocratie atlantique - un monde de valeurs et d'économies libérales qui tirait en partie sa force idéologique du contexte de la Guerre froide - et l'histoire globale ou atlantique telles qu'elles sont pratiquées aujourd'hui. Alors que l'approche de Palmer et de Godechot visait à expliquer les révolutions politiques et l'émergence de la démocratie en les plaçant au centre d'une histoire internationale, l'intérêt actuel pour l'histoire transnationale et globale a des origines tout à fait différentes. Il est issu, comme cela vient d'être souligné, de l'histoire coloniale et impériale. Le point commun des deux perspectives, toutefois, c'est le désir de ne pas rester confiné dans l'histoire nationale et dans les cadres narratifs que celle-ci a engendrés.
\end{abstract}

\title{
Matthias Middell
}

Le nouvel intérêt à l'égard de l'histoire transnationale ou globale a d'autres origines encore : je pense aux travaux d'un groupe d'historiens des années 1950 et 1960 sur les « jacobins extra muros». Un débat sur l'éphémère république de Mayence a commencé dans le contexte de la Guerre froide mais l'attention s'est vite portée sur l'ensemble des jacobins qu'on découvrait de Hambourg à Vienne ou Budapest, et aux conséquences extra-européennes de la Révolution-comme les mouvements indépendantistes d'Amérique latine. Ceci est lié à deux événements contemporains. D'une part la décolonisation en Afrique et en Asie à partir de la fin des années 1950. Celle-ci ne s'intégrait pas à la théorie trop simple de la modernisation, ni à celle du passage mondial imminent au socialisme ou au communisme. Car si les révolutions peuvent s'exporter, combien de partisans d'une transformation radicale faut-il pour que le bacille puisse se développer? Et comment fonctionne la communication entre des révolutions qui se déclenchent au même moment en différents lieux ? La Révolution était encore considérée comme un réservoir d'exemples pour trouver par analogie des réponses aux questions contemporaines.

D'autre part, dans le bloc de l'Est, les partis communistes ont présenté les sociétés qu'ils dominaient comme le résultat d'une rupture révolutionnaire. En fait cette rupture n'a pas été la conséquence d'une révolution mais a eu lieu sous la pression des baïonnettes de l'armée rouge. Est-ce que les jacobins hors de France n'avaient pas été dans une telle situation : trop faibles pour bouleverser leur propre société et pour prendre 
le pouvoir, et pour cette raison obligés de compter sur la protection militaire des révolutionnaires français?

Ceci a donné lieu à une très riche littérature qui impressionne souvent par l'ampleur des sources mobilisées. Pour réfléchir à cette question d'une histoire mondiale des révolutions, la perspective n'était pas simplement transatlantique : on cherchait explicitement à dépasser l'eurocentrisme traditionnel ${ }^{7}$. Pourtant, malgré les nombreuses coopérations et sympathies à gauche, cette pratique n'a exercé qu'une influence marginale sur l'historiographie française (et anglo-américaine).

On peut dire la même chose des origines de l'histoire connectée. Il est vrai que pendant longtemps le paradigme comparatiste, souvent lié à des concepts diffusionnistes, a dominé l'historiographie. Mais il faut rappeler que Marc Bloch, déjà en 1928, mettait en garde contre un usage naïf de la comparaison et soulevait le problème de la mutabilité des éléments à comparer. Ceci a été pris en compte dans les études sur les transferts culturels que Michel Espagne et Michael Werner ont promues. Donc on ne peut pas dire que l'idée d'histoire connectée n'a pas touché la France, bien au contraire.

\section{Karine Rance}

Êtes-vous prêts à suivre David Armitage lorsqu'il écrit en plaisantant : «We are all Atlanticists now $»^{8}$ ? Est-ce que l'histoire atlantique, telle qu'elle se pratique aujourd'hui en Grande Bretagne ou aux États-Unis a encore à voir avec celle de Robert Palmer et Jacques Godechot ? Certains regrettent que cette histoire soit d'abord celle d'une Atlantique américaine, au mieux celle d'une Atlantique britannique, et finalement assez peu le lieu d'une pratique scientifique à vocation transnationale.

\section{Lynn Hunt}

Le modèle atlantique actuel n'est pas celui de Palmer et de Godechot car l'accent est mis sur l'esclavage et sur les connexions entre la France, l'Afrique et les Caraïbes, plutôt que sur la démocratie et sur les connexions entre les patriotes français, américains et britanniques, les nationalistes irlandais, ou les Belges, Néerlandais, Allemands, italiens et Polonais qui ont combattu pour des constitutions et la participation populaire.

(7) Voir Manfred KossoK, In Tyrannos. Revolutionen der Weltgeschichte, Leipzig, 1989. (8) David ARMiTAGE, «Three concepts of Atlantic History», dans David ARMITAGE, Michael J. BRADDICK (dir.), The British Atlantic World 1500-1800, New York, Palgrave Macmillan, 2002, p.11-27, ici p. 11. 
Le modèle atlantique attire l'attention sur le rôle de la France dans le commerce des esclaves et dans les circuits monétaires, d'esclaves et de biens reliant (plutôt lâchement) les différentes côtes atlantiques. S'il est indubitable que la question de l'esclavage est mêlée à la Révolution française, on est encore loin de savoir comment l'esclavage a pesé sur le déroulement des événements révolutionnaires au sein de la France métropolitaine, et même s'il a pesé d'une manière déterminante (malgré les efforts de Popkin, Dubois et Régent pour affirmer que cela a été le cas). De plus, on ne peut pas se limiter au monde atlantique car la traite des esclaves dépendait d'une manière non négligeable des textiles d'Asie du sud et des cauris. De ce fait, ce trafic était intimement lié aussi aux échanges de l'Océan indien. La traite des esclaves était un phénomène global au XVIII ${ }^{\mathrm{e}}$ siècle, pas simplement atlantique. Il reste donc beaucoup à savoir sur l'impact de l'esclavage sur la France, à la fois avant et pendant la Révolution française, et plus généralement sur le rôle de l'esclavage dans l'économie française globale.

\section{Matthias Middell}

Dans le courant relativement neuf de l'histoire globale, des historiens continuent à se concentrer sur l'histoire atlantique. Mais pour différentes raisons, la prise en compte de la relation avec l'Asie est de plus en plus souvent considérée comme indispensable.

En Allemagne, la tendance est à l'ouverture de l'histoire nationale à l'histoire européenne en faisant souvent appel au paradigme de l'histoire transnationale. Les atlantistes qui travaillent sur les liens entre l'Europe et l'Amérique en revanche n'y sont pas très nombreux.

\section{Paul Cheney}

Un certain nombre de tendances qui étaient occultées par le révisionnisme, l'anti-révisionnisme et le post-révisionnisme sont apparues clairement après que l'orage soit calmé, à la fin des années 1990 (une tempête dans un verre d'eau !). D'abord, l'arrivée au pouvoir en 1981 de François Mitterrand et de Ronald Reagan a séparé les chemins politiques de la France et des États-Unis. Cela a porté atteinte à la prétendue solidarité politique entre les deux pays qui avait nourri la théorie palmériste des révolutions atlantiques franco-centrées. L'incident diplomatique à propos de la guerre en Iraq n'a fait que renforcer cette impression de rupture. Les Américains de gauche peuvent certes échapper temporairement à l'hégémonie néo-libérale en partant en exil en France pour des séjours de recherche, ou apprécier le confort froid d'un exil intérieur dans leur environnement 
universitaire mais la connexion entre les traditions politiques française et américaine, connexion qui assurait un lectorat et des étudiants aux spécialistes de la Révolution française, n'est plus évidente. De nouvelles connexions entre des traductions devaient être établies.

Alors que la solidarité franco-américaine devenait moins évidente, l'atlantisme politique des ères Thatcher/Reagan et Blair/Clinton-Bush faisait fructifier l'histoire atlantique sous une forme spécifiquement angloaméricaine. Le séminaire d'histoire atlantique de Bernard Bailyn (19962010) a porté largement sur les empires espagnol et français, et Bailyn lui-même a consacré une large place au cadre impérial dans son essai (qui a fait date) sur l'histoire atlantique (2005). Cependant le programme de ces séminaires - un indicateur presque parfait de la production de monographies sur l'histoire atlantique - était dominé par l'expérience anglo-américaine. Je ne veux pas dire que la polarisation de l'attention sur le monde atlantique anglo-américain indique une sympathie des universitaires à l'égard du néo-libéralisme ou de la «Troisième Voie » clintonienne/blairiste, mais il me semble évident que la fortune de l'atlantisme à l'Université est liée d'une certaine manière à l'atlantisme politique contemporain.

À la même époque, la nouvelle histoire impériale britannique a participé au renouvellement de l'histoire moderne britannique - et lui a donné une perspective plus globale. Enfin, c'est à cette époque aussi que les Subaltern Studies, concentrées sur l'histoire de l'Asie du Sud et sur les problèmes spécifiques du colonialisme et du post-colonialisme britannique, ont commencé à s'imposer, proposant une autre forme d'histoire connectée à l'intérieur de l'Empire britannique et au-delà? .

\section{Alan Forrest}

L'histoire atlantique, telle qu'elle se développe actuellement, est en effet très différente de ce qui s'est fait dans les années 1960 : elle ne se concentre plus sur les seuls liens entre l'Europe et l'Amérique du Nord mais s'étend à d'autres cultures pour intégrer le monde non-européen. La traite des esclaves était l'une des connexions majeures qui se sont développées à l'époque moderne entre l'Europe et l'Afrique, ce qui en fait un objet d'étude particulièrement intéressant de ce point de vue. Cette évolution a aussi coïncidé avec un changement dans la manière dont la traite des esclaves a été étudiée, avec plus d'importance donnée à l'expérience des

(9) Dipesh Chakrabarty, Provincializing Europe: Postcolonial Thought and Historical Difference, Princeton, Princeton University Press, 2000. 
esclaves en Afrique et pendant le passage aux Amériques, à leur politisation sur les plantations, et à ses conséquences. Le poids du «présentisme » est clair ici. L'esclavage est un domaine qui se rattache aux questions politiques clés de notre temps, du genre et de l'ethnicité aux migrations, qui, dans les trente dernières années, ont été particulièrement débattues. Il est d'ailleurs impossible d'enseigner dans une université hors de France sans être conscient de l'étendue de ces changements. Les nouveaux postes sont souvent rattachés à l'histoire impériale ou globale (et pas seulement atlantique : l'Inde et plus largement l'Asie sont l'objet d'un intérêt de plus en plus marqué). Cela signifie que la Révolution française, dans son contexte européen, risque de perdre sa position privilégiée comme moment clé dans la formation du monde moderne.

\title{
Karine Rance
}

Le maintien du cadre national dans l'étude de la Révolution française ne tient-il pas à la difficulté qu'il y a à articuler cette histoire - souvent politique - à une échelle supra-nationale $?^{10}$

\begin{abstract}
Alan Forrest
Je pense que la situation est en train de changer. Il est vrai que certains historiens de l'école classique (ou marxiste) ont insisté sur le fait que la Révolution était un mouvement spécifique à la France, sa culture, et sa tradition républicaine. L'identification de la Révolution avec la République y a contribué. Cela explique l'insistance avec laquelle en France on regarde à travers le prisme de l'histoire nationale et les difficultés qu'ont eues les spécialistes de la Révolution française à inscrire leur sujet dans la longue durée. Il est significatif de voir que certaines des tentatives les plus radicales faites par des universitaires étrangers pour relativiser la Révolution française viennent des sciences sociales plus que des facultés d'histoire : soit des théoriciens politiques du XIX ${ }^{\mathrm{e}}$ siècle comme Marx et Max Weber, soit des sociologues du XX $\mathrm{X}^{\mathrm{e}}$ siècle comme Barrington Moore et Theda Skocpol, dont les travaux ont été adaptés pour proposer des théories de la révolution ou pour rechercher les modalités du passage dans la modernité. Déjà dans les années 1960, Moore regardait au-delà de l'Europe (et des comparaisons habituelles entre les révolutions anglaises, française, américaine et russe) pour inclure les puissances asiatiques comme la Chine et l'Inde.
\end{abstract}

(10) Bernhard STRUCK, Kate FERRIS, Jacques REVEL, «Introduction: space and scale in transnational history», The International History Review, 33, 4, 2011, p. 573-584. 


\section{Matthias Middell}

En fait de nombreuses études n'ont pas pour cadre l'espace national mais portent sur une région particulière. Elles suivent souvent le modèle de la « nation en petit », pour montrer que tous les événements significatifs qui se déroulaient à Paris avaient aussi lieu dans les provinces les plus éloignées, ou au moins y trouvaient un écho. La question théorique qui se pose pourtant - et elle est passionnante - est de savoir s'il n'y a pas eu en France différentes voies de transformations socio-économiques et socio-culturelles. Je rappellerai la tentative d'Anatoli Ado pour arriver à une synthèse des rythmes, des contextes et des revendications des mouvements paysans entre 1789 et 1794, à partir des nombreuses monographies régionales disponibles ${ }^{11}$. L'Atlas de la Révolution française relève de la même démarche, tout comme l'ouvrage de Michel Vovelle qui pose la question d'une géopolitique spécifique de la Révolution ${ }^{12}$. On voit une France qui se consolide comme État territorial et une autre France des réseaux commerciaux qui a des ramifications sur de longues distances - pensons à Nantes, Bordeaux ou Marseille. On ne s'étonne guère que dans ces lieux se trouve une «mémoire de l'étranger $»^{13}$. La Révolution française perd peut-être ainsi son statut d'exception et de domaine d'enseignement sui generis, et se dilue dans une histoire à plus grande échelle. Dans un pays comme l'Allemagne où, dans le passé, il était impossible de faire une carrière universitaire en ne travaillant que sur la Révolution française, on aura moins le sentiment d'avoir perdu quelque chose que dans des pays où elle reste un domaine d'enseignement à part entière.

\section{Paul Cheney}

Comme je l'ai dit ailleurs ${ }^{14}$, le marxisme et la théorie de la modernisation libérale se sont tous deux concentrés sur le processus de modernisation politique et économique au sein de l'État-nation, ce qui rejoint l'idée dominante au $\mathrm{XX}^{\mathrm{e}}$ siècle du lien entre développement économique et formes de gouvernement à l'époque de la politique de masse. Loin de relativiser cette approche orientée sur l'État-nation, les comparatistes qui observent

(11) Anatoli V. ADO, Paysans en Révolution : terre, pouvoir et jacquerie, 1789-1794, Paris, 1996 (Moscou 1971, 1986).

(12) Michel Vovelle, La découverte de la Politique. Géopolitique de la Révolution française, Paris, 1993.

(13) Michel Espagne, Bordeaux Baltique. La présence culturelle allemande à Bordeaux aux XVIII et XIX ${ }^{e}$ siècles, Paris, 1991.

(14) Paul CHENEY, op. cit., p. 14-20. 
l'expérience révolutionnaire pour expliquer le développement de la démocratie libérale ou de l'autoritarisme fasciste et communiste tendent à le renforcer ${ }^{15}$.

Le révisionnisme, après les années 1970, a gardé pratiquement intacte la structure nationale/comparatiste de l'histoire de la Révolution française pour plusieurs raisons. Parmi les révisionnistes comme François Furet et Ran Halevi, le libéralisme anglo-américain, adopté par les révolutions relativement modérées de 1689 et 1776, était érigé en norme à partir de laquelle les excès de la Terreur pouvaient être expliqués et jugés. Le modèle de culture politique développé par Keith Baker n'était pas dénué de référence implicite à une relation plus saine entre l'État et la société civile en Grande Bretagne. Mais dans l'ensemble les travaux entrepris par les figures dominantes dans ce domaine étaient plutôt orientés sur l'histoire nationale. Ceci était en partie lié au prestige de l'historiographie française des années 1950, jusqu'au début des années 1990. Mais comme Bill Sewell l'a affirmé16, le tournant culturel et linguistique fut aussi le produit d'une tendance prospère et complaisante, pendant les années Reagan/Bush1/Clinton à récuser l'importance, soulignée avant par les marxistes, des évolutions matérielles ; les historiens qui ont pris le tournant culturel et linguistique ont repoussé à l'arrière-plan les transformations sociales et économiques qui intéressent de nombreux spécialistes de l'histoire globale ou impériale. Les écrits récents sur la France globale donnent souvent l'impression de vouloir rattraper le temps perdu.

\section{Karine Rance}

Lorsque des études sur la Révolution française dépassent le cadre national, elles se placent souvent dans une logique diffusionniste : on observe la propagation, à partir du foyer français, des idées et modèles politiques en Europe - et depuis quelques années dans les colonies. Peut-on dépasser cette perspective en tenant compte, comme Bailey Stone le proposait, des influences extérieures (contexte international économique, commercial, financier et diplomatique) dans le processus

(15) Barrington MOORE JR, Social Origins of Dictatorship and Democracy. Lord and Peasant in the Making of the Modern World, Boston, Beacon Press, 1967 ; Jack GOLDSTONE, Revolution and rebellion in the Early Modern World, Berkely, University of California Press, 1991 ; Thecla SKOCPOL, States and Social Revolutions : A comparative Analysis of France, Russia and China, Cambridge University Press, 1979.

(16) Bill SEWELL, « The Political Unconscious of Social and Cultural History, or Confessions of a Former Quantitative Historian », in Logics of History: Social Theory and Social Transformation, Chicago, University of Chicago Press, 2005, p. 22-80. 
de radicalisation révolutionnaire ${ }^{17}$ ? Il est vrai qu'on a reproché à sa démonstration d'aboutir paradoxalement à renforcer l'homogénéité d'une nation française présupposée et le poids de l'État...

\section{Matthias Middell}

Un des problèmes de la perspective diffusionniste est que souvent on s'arrête au constat de l'existence ou de l'écho d'un phénomène dans un lieu éloigné de son origine supposée. L'étude de Rolf Reichardt et HansJürgen Lüsebrink sur les transferts culturels entre la France et les territoires allemands entre 1770 et 1820 a apporté de tout autres résultats ${ }^{18}$. Ils ont considéré l'ensemble des traductions de textes français qui circulaient dans les États allemands et cherché quel en était le sujet. Étonnamment, la politique se situe loin derrière les questions de société et les « connaissances utiles ». La Révolution a donc certes déclenché une vague d'intérêt à l'égard de la France, mais cet intérêt concernait tout autre chose que ce que des générations d'historiens du politique avaient supposé. Alors si on retourne la question pour se demander ce qui est véritablement significatif dans l'intégration d'un phénomène dans une culture ou une société, on obtient des réponses très différentes.

Ceci pourrait d'ailleurs mener à une autre piste de recherche qui est évoquée dans le volume de Armitage et Subrahmanyam (2010) : on a longtemps vu la Révolution française comme le début d'une nouvelle époque, ce qui conduit automatiquement à chercher ce que les générations suivantes ont fait de cette expérience révolutionnaire dans d'autres pays (une question qui, pour des raisons similaires, a intéressé autant les libéraux que les conservateurs, ou Marx et ses partisans). Mais on peut renverser la perspective et voir les révolutions en Amérique du Nord, en France, puis dans les Caraïbes et en Amérique du Sud comme la fin d'une longue chaîne de tentatives avortées pour se soustraire à l'emprise des empires ou pour les moderniser. Il faudrait dans ce cas se demander quelles leçons les acteurs de la Révolution française ont apprises en regardant vers l'Est et vers le Sud.

(17) Bailey Stone, Reinterpreting the French Revolution : A Global-Historical Perspective, Cambridge, Cambridge University Press, 2002.

(18) Hans-Jürgen LÜSEBRINK, Rolf REICHARDT (dir), Kulturtransfer im Epochenbruch. Frankreich-Deutschland 1770 bis 1815, 2 vols, Leipzig, 1997. 


\section{Lynn Hunt}

Si un domaine n'a jamais été négligé c'est bien celui de l'influence de la Révolution française. Les contemporains des événements ont été immédiatement stupéfaits par les bouleversements provoqués par les idées et les pratiques révolutionnaires (Burke, Paine, Wollstonecraft) et ont ensuite été les témoins de l'impact considérable des guerres révolutionnaires et napoléoniennes en Europe, en Afrique du Nord, et dans les nouveaux ÉtatsUnis (achat de la Louisiane), et finalement dans toute l'Amérique latine (elle a obtenu son indépendance grâce à Napoléon qui avait sapé l'autorité des dirigeants espagnols et portugais). Il n'est donc guère surprenant que les historiens aient toujours été attentifs à la dimension globale de la Révolution française. Pourtant ils ne se sont tournés que récemment vers les effets de l'empire global et de la compétition impériale globale sur la Révolution elle-même.

Le modèle impérial global n'est pas totalement mis en œuvre car les historiens tendent à se concentrer sur un empire, en excluant les autres sauf s'ils entrent en conflit, et orientant presque toute leur attention sur l'histoire diplomatique et militaire. Il est regrettable que le modèle impérial reste aussi peu développé en France et il y a beaucoup à gagner à considérer la Révolution française comme un épisode de l'histoire de France en tant qu'Empire. Bailey Stone (2002), par exemple, ne propose pratiquement aucune analyse de la France comme empire colonial. Quand le terme apparaît, il renvoie à l'histoire du Saint-Empire, de l'Empire Ottoman ou de l'Empire napoléonien. Une raison majeure de cette négligence est suggérée par Paul Cheney (2010), qui affirme que l'empire était rarement un sujet de discussion en France avant 1789.

\section{Paul Cheney}

Cécile Vidal a évoqué de manière très convaincante et documentée la « réticence des historiens français à l'égard de l'histoire atlantique ${ }^{19}$ mais cette réticence a été largement surmontée entre-temps. Le changement me semble assez soudain même s'il y avait des précédents évidents avec les travaux notamment de Marcel Dorigny, Yves Bénot et Bernard Gainot. 


\section{Alan Forrest}

L'historiographie française change actuellement rapidement dans ce domaine, mais les travaux sur l'Empire portent essentiellement sur l'Empire britannique et sur le monde anglo-saxon, où la Révolution française a joué un rôle plus périphérique. On s'est surtout intéressé à la diplomatie, au commerce et à la guerre, plus qu'à la circulation des idées ; ceci révèle clairement un monde interdépendant, même si généralement Londres en reste le cœur, en tant que centre politique et commercial de l'Empire.

La dimension globale de la Révolution française a depuis longtemps été un thème d'analyse historique, mais d'une manière qui s'accorde difficilement avec un aspect majeur de l'histoire globale actuelle. Celle-ci insiste en effet sur la réciprocité, la circulation des gens, des biens et des idées entre les cultures, depuis les régions marginales de l'Empire vers la capitale, autant que dans le sens inverse. La Révolution française est traditionnellement vue comme un mouvement des idées à sens unique (du moins hors d'Europe), voire explicitement comme un don de liberté fait par la France aux peuples du monde, arrivant dans les Caraïbes via les décrets de la Convention ou les lettres des agents de Bordeaux ou de Nantes. C'est aussi de cette manière que les artistes contemporains l'ont représentée.

\section{Karine Rance}

La « réticence française » à l'égard du global turn est aussi liée à la « focalisation franco-française » croissante de la recherche historique en France qui a été dénoncée par Caroline Douki et Philippe Minard ${ }^{20}$. Celle-ci a été expliquée par des raisons institutionnelles (liées à la fonction civique de l'enseignement de l'histoire en France) autant que politiques : refoulement du passé colonial et articulation spécifique de la nation à la République. S'ajoute pour la période révolutionnaire la question de l'exception française.

Lynn Hunt regrettait ailleurs ${ }^{21}$ que la France n'ait pas encore produit d'ouvrage équivalent à The ideological origins of the British Empire de David Armitage : un ouvrage qui tiendrait compte du contexte global et de l'impact des colonies (depuis l'influence de lobbies défendant des intérêts privés dans les différentes assemblées jusqu'aux événements qui s'y déroulent). Est-ce que ce serait véritablement imaginable dans la mesure

(20) Caroline DoukI et Philippe MinARD, « Histoire globale, histoires connectées : un changement d'échelle historiographique ? », RHMC, 2007/ 5, p. 7-21.

(21) Lynn HunT, «The French Revolution in Global Context», dans Armitage SUBRAHMANYAM, op. cit. 
où, comme Paul Cheney l'écrit, la notion d'Empire n'a pas du tout le même sens en France et outre-Manche ? Cela paraît d'autant plus difficile que, depuis le $\mathrm{XIX}^{\mathrm{e}}$ siècle, le récit national français est républicain avant d'être impérial, comme en attestent Les Lieux de mémoire de Pierre Nora (avec une seule entrée sur le fait colonial ${ }^{22}$. Qu'est-ce que la notion d'Empire peut apporter à notre compréhension de la Révolution française ?

\section{Paul Cheney}

La notion d'empire n'a pris vraiment d'importance en France qu'une fois qu'elle n'était plus l'un des nombreux concepts vagues pour désigner les éléments d'une monarchie composite. L'empire n'était pas « idéologique » (au sens où l'entend Armitage) en France jusqu'à ce qu'il devienne, dans les années 1780, un objet de comparaison dans une perspective critique et de réforme. Il y a un récit important à construire à propos de ce processus, mais ce serait une erreur, je pense, d'exagérer l'importance de l'empire dans l'identité nationale française.

L'histoire connectée pratiquée par Sanjay Subrahmanyam explore les phénomènes du métissage culturel, des formes impériales de gouvernance et de commerce s'influençant mutuellement, souvent par l'étude de la circulation des textes et des individus à travers l'espace impérial. Un objectif majeur de ce type d'histoire est d'explorer les distinctions centrepériphérie qui structurent l'histoire globale et impériale, souvent sous l'influence de la théorie du système monde de Wallerstein.

L'histoire connectée ouvre de nouvelles perspectives sur l'histoire impériale et océanique, mais je partage les réserves de chercheurs comme Trevor Burnard ${ }^{23}$ et Jean-Paul Zuniga ${ }^{24}$ : les empires modernes étaient organisés autour d'un centre métropolitain, même si le centre ne contrôlait pas la périphérie autant que les administrateurs et les marchands l'auraient voulu. La notion de connexion implique un réseau et, dans tout réseau les connexions sont plus ou moins denses en certains lieux, renforçant le pouvoir des individus et des institutions qui les organisent. Une vision excessivement post-moderniste, foucaldienne de l'Empire fait courir aux historiens le risque de perdre le sens de la réalité du pouvoir.

(22) Jean-Frédéric SCHAUB, "La catégorie "études coloniales" est-elle indispensable ? », Annales $H S S$, mai-juin 2008, n³, p. 625-646.

(23) Trevor BURNARD, «Empire Matters ? The Historiography of Imperialism in Early America, 1492-1830 », History of European Ideas, 2007, 33, p. 87-107.

(24) Jean-Paul ZuNIGA, «L'Histoire impériale à l'heure de l' "histoire globale" », Revue d'histoire moderne et contemporaine, 2007, $\mathrm{n}^{\circ}$ 54-4bis (5) (December), p. 54-68. 


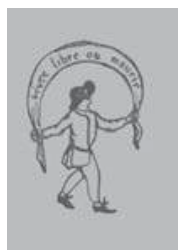

\section{Lynn Hunt}

Pour Paul Cheney (2010) l'empire est en réalité nécessaire pour entrer dans la compétition du commerce global du XVIII ${ }^{\mathrm{e}}$ siècle, ce n'est pas un facteur autonome. Cheney concentre plus son analyse sur l'Ancien Régime que sur la Révolution et évoque une « globalisation archaïque » qui couvre le XVIII ${ }^{\mathrm{e}}$ siècle. Mais au moins il met la question de la globalisation au premier plan, en affirmant qu' une monarchie constitutionnelle réformiste ne pouvait pas émerger car les gouvernants restaient divisés sur le rôle du commerce et incapables pour cela de répondre aux défis posés par la rivalité du commerce britannique. Il est surprenant, de ce point de vue, que le travail de Guillaume Daudin (Commerce et prospérité, 2005) qui analyse le rôle du commerce extra-européen au sein de l'économie française du XVIII ${ }^{\mathrm{e}}$ siècle reste aussi peu commenté par les historiens de la Révolution française, y compris Paul Cheney ${ }^{25}$. C'est un point de départ essentiel pour toute recherche sur la globalisation au XVIII ${ }^{\mathrm{e}}$ siècle.

\section{Paul Cheney}

Les historiens de la Révolution française interrogent maintenant la manière dont l'évolution du monde capitaliste a été modifiée à l'époque de la Révolution, et osent même revoir - d'une manière que le révisionnisme antimarxiste avait rendue taboue - comment l'évolution du capitalisme moderne pourrait avoir provoqué la Révolution ${ }^{26}$. L'histoire du capitalisme a toujours entretenu une relation complexe avec l'histoire des événements politiques comme la Révolution française, même si on étire la Révolution de 1789 à 1815. En effet, l'une des critiques les plus fortes de François Furet à l'égard de ce qu'il appelait la tradition jacobino-marxiste, était que ses partisans croyaient que de profonds changements sociaux pouvaient avoir lieu en l'espace d'une ou deux décennies. Ce reproche pourrait être plus valide dans le contexte métropolitain que dans le contexte colonial. L'histoire globale du capitalisme et de la Révolution française - conçue dans sa dimension atlantique, impériale - peut aider les historiens à repenser les temporalités de la transformation de l'économie capitaliste. Dans des lieux comme La Havane, Saint-Domingue, la Jamaïque, la Guadeloupe, la Martinique, le Brésil et Rio de la Plata, des bouleversements dans l'économie de plantation ont provoqué des transformations sociales, économiques et environnementales rapides. Des lieux plus institués, moins exposés (ou 
moins dynamiques) comme la France rurale ont changé plus lentement. Une discussion comparative de ces changements aide à mesurer l'importance, dans le processus de développement capitaliste, des structures légales, politiques et sociales préexistantes. Une comparaison des révoltes rurales dans les campagnes françaises avec celles des colonies esclavagistes - et la réaction des élites - ouvre de nouvelles possibilités de comparaisons entre les provinces de l'intérieur et de l'extérieur.

\section{Alan Forrest}

La force du lobbying au cours des premiers mois de la Révolution, à la fois par les chambres de commerce et par les planteurs dans les îles elles-mêmes, témoigne du poids des intérêts coloniaux. La voix des planteurs s'exprimait à Paris au début de la Révolution à travers le club Massiac et différents groupes d'intérêt. Cela dit, la place de l'Empire dans la conscience nationale était certainement plus forte en Grande Bretagne qu'en France, ce qui explique en partie que les historiens britanniques du XVIII ${ }^{\mathrm{e}}$ siècle soient plus conscients du rôle déterminant joué par le commerce atlantique. La Grande Bretagne a toujours davantage dépendu de la mer pour sa richesse, et à la fin du XVIII e siècle, l'Empire était devenu si central pour son identité, offrant un pôle d'unité aux nations qui constituent les Îles britanniques. Les banques et les assurances maritimes étaient bien plus développées en Grande Bretagne aussi, et Londres avait une présence financière bien plus forte que Paris. Cela signifie que les colonies et l'économie étaient très étroitement imbriquées, plus, je pense, qu'en France. À partir de la guerre de Sept Ans - une guerre qui commence dans les Amériques - les conflits entre les deux pays ont une dimension coloniale décisive. Et ces rivalités coloniales signifiaient que la Grande Bretagne serait toujours l'adversaire de la France dans l'espace extra-européen. Le commerce colonial et la capacité militaire étaient inextricablement liés.

\section{Matthias Middell}

Le cadre de l'État-nation reste tout à fait important mais il n'est plus le seul et il n'est probablement pas non plus l'instrument le plus efficace pour appréhender le défi global. Ceci a donné lieu à un large débat sur les nouvelles formes de la territorialisation et de la mise en réseau, autour de concepts comme le «new regionalism » et les «transnational value chains ». À partir de ce débat sur le début et la fin d'une époque de la 
territorialité ${ }^{27}$ se pose la question de savoir quel régime de territorialisation existait avant l'État-nation et quelles formes de contrôle des courants globaux sont liées au concept d'Empire. La Révolution française, de ce point de vue, peut être interprétée comme une tentative pour libérer la société de ces structures impériales afin de réagir plus efficacement à la globalisation. D'ailleurs le traité de Vienne n'établit pas un monde d'Étatsnations : il ouvre en Europe une longue période de transition marquée par la coexistence entre États-nations et Empires.

\section{Karine Rance}

Les recherches se sont multipliées - et on ne peut que s'en féliciter sur les espaces coloniaux et sur des interactions révolutionnaires parfois très éloignées de l'hexagone ${ }^{28}$. Comment expliquer, après un si long silence, l'engouement actuel à l'égard de la question coloniale ?

\section{Lynn Hunt}

Depuis que Michel-Rolph Trouillot a affirmé il y a une vingtaine d'années que la révolution haïtienne avait été systématiquement supprimée de l'historiographie occidentale ${ }^{29}$, cette révolution réprimée s'est vengée en devenant le sujet de nombreuses études de premier ordre. L'intérêt pour la révolution des esclaves s'est d'abord imposé dans les Caraïbes ${ }^{30}$, puis aux États-Unis (avec John Garrigus, Laurent Dubois et Jeremy Popkin entre autres) avant de revenir assez lentement en France. Le travail de Trouillot est devenu le sujet d'une discussion soutenue en France vers 2010 et n'a jamais été cité dans les $A H R F^{31}$. Toutefois, grâce aux travaux pionniers de Marcel Dorigny, Florence Gauthier, Bernard Gainot et Frédéric Régent, l'histoire de la traite française et de la colonisation dans les Caraïbes fait maintenant en France aussi pleinement partie de l'histoire de la Révolution française.

(27) Charles MAIER, « Consigning the Twentieth Century to History : Alternative Narratives for the Modern Era », The American Historical Review 105 (2000), n³, p. 807-831.

(28) Rachel Hope ClEves, " "La terreur des esclavagistes" : la Révolution française et les origines de l'abolitionnisme en Amérique », AHRF n 363, 2011, p. 85-107, numéro spécial : L'Amérique du Nord à l'époque de la Révolution française, coordonné par Carla Hesse et Timothy Tackett.

1995.

(29) Michel-Rolph Trouillot, Silencing the Past: Power and the Production of History,

(30) Cyril Lionel Roberet JAMES, The Black Jacobins, 1938.

(31) Je dis ceci sur la base d'une recherche menée sur CAIRN. Deux discussions antérieures en français (Frederick Cooper en 2001 et Ada Ferrer en 2003) sont le fait de deux historiens américains. 


\section{Paul Cheney}

La raison de ce regain d'intérêt, du moins dans le contexte NordAméricain où la production scientifique sur la révolution haïtienne est considérable, n'est pas terriblement compliquée : les historiens américains, comme la société américaine dans son ensemble, sont préoccupés par les questions de race et d'identité. Une histoire de la Révolution française réorganisée autour de l'esclavage, de son abolition et de l'exclusion du projet révolutionnaire des minorités raciales, ethniques ou religieuses coïncide parfaitement avec ces préoccupations. Les universitaires français se sont plus intéressés au passé colonial de la France quand les problèmes d'immigration, de race, de citoyenneté et d'assimilation ont occupé une place plus centrale dans le débat politique. Une Révolution française globalisée - mais dans ce cas atlantique - a pris davantage sens en France comme aux États-Unis.

\section{Alan Forrest}

Haïti était important, non seulement comme preuve des contradictions internes de l'idéologie révolutionnaire (est-ce que les esclaves et les libres de couleur peuvent bénéficier également des droits de l'homme? Est-ce que le droit de propriété des possesseurs d'esclaves peut être légitimement bafoué ?), mais aussi comme balise d'espoir et modèle pour d'autres nations et d'autres peuples colonisés, particulièrement en Amérique. En fusionnant les idées des droits de l'homme et de l'anti-colonialisme, Haïti a forgé une idéologie qui était spécifique aux besoins et aux aspirations de la plupart du Tiers-monde. Les historiens ont débattu vigoureusement sur l'ampleur de la dette envers la France. Mais est-ce que la responsabilité des rébellions des esclaves qui ont secoué l'île revient véritablement aux hommes politiques à Paris ? Ou est-ce que l'agency doit être donnée aux esclaves eux-mêmes, le soulèvement étant le produit de leur expérience dans les champs de canne ? Cette théorie a été défendue il y a déjà bien longtemps, dans les années 1930, par Cyril Lionel Robert James qui affirmait que le travail dans les plantations de sucre donnait aux esclaves un sens de classe qui était même plus important que la race dans la formation de leur identité.

\section{Matthias Middell}

Les excellentes études sur la Révolution haïtienne ont sans aucun doute transformé profondément le paysage historiographique. Ceci est lié aux débats sur les questions de race et d'esclavage aux États-Unis et au 
regard français sur la diversité des situations coloniales. Du côté allemand, Michael Zeuske a contribué aux recherches sur les transformations de l'économie de plantation de Haïti à Cuba entre 1795 et 1880 et sur les conséquences socio-culturelles de ces transformations dans les centres de l'ancien marché mondial du sucre. Haïti n'est pas seulement importante pour comprendre le processus de radicalisation en France mais aussi pour savoir à qui revient l'initiative de l'abolition de l'esclavage et quelles en ont été les conséquences sur les structures des colonies de monoculture. Il apparaît ainsi que l'élargissement du focus géographique de l'historiographie révolutionnaire permet de relier la Révolution française avec des processus de longue durée, notamment avec l'histoire du sousdéveloppement post-colonial.

\section{Karine Rance}

Pensez-vous qu'il faille placer les colonies au cœur du processus révolutionnaire français?

\section{Paul Cheney}

Oui mais dans certaines limites. Une histoire globale ou connectée de la Révolution française doit relativiser quelque peu l'importance de la race et de l'esclavage pour la Révolution dans son ensemble, et aussi prendre explicitement en compte l'évolution du système capitaliste dans ce que Christopher A. Bayly a nommé la phase finale de la globalisation archaïque. Ces phénomènes sont évidemment intimement connectés, donc ce qui est en question ici c'est de découvrir quelles sont ces connexions.

Indiscutablement, les soulèvements des esclaves et l'agitation politique des libres de couleur ont été décisifs et ont intensifié l'impact de la Révolution française dans toutes les colonies françaises esclavagistes. Comme David Geggus l'a montré ${ }^{32}$, l'esclavage, les soulèvements d'esclaves et la menace de l'émancipation sont entrés dans les calculs stratégiques des grandes puissances dans le monde atlantique. Les travaux de John Thornton ${ }^{33}$ et Malick Ghachem ${ }^{34}$ ont fourni d'excellents exemples

(32) David Patrick GEGGUS, « Slavery, War and Revolution in the Greater Caribbean, 17891815 », dans A Turbulent Time: The French Revolution and the Greater Caribbean, Bloomington, Indiana University Press, 1997, p. 1 - 50 ; Haitian Revolutionary Studies, Bloomington, Indiana, Indiana University Press, 2002.

(33) John K. THORNTON, "I Am the Subject of the King of Congo": African Political Ideology and the Haitian Revolution, » Journal of World History 4 (2), 1993 (October 1), p. 181-214. (34) Malick W. GHACHEM, The Old Regime and the Haitian Revolution, Cambridge, New York, Cambridge University Press, 2012. 
d'histoire connectée de la Révolution française, ancrée dans le problème de la race et de l'esclavage, montrant par exemple l'influence de la pensée politique africaine sur le royaume pendant le soulèvement des esclaves de Saint-Domingue, ou les usages étonnamment radicaux que les esclaves et partisans de l'abolition ont fait de la politique absolutiste (le Code Noir). Ce qu'il faut éviter, à mon avis, ce sont les positions excessives de chercheurs comme Laurent Dubois, qui affirme que les révoltes des esclaves ont eu un effet fondamental et déterminant sur l'idéologie révolutionnaire à l'intérieur de la France ${ }^{35}$. Jeremy Popkin va trop loin dans la direction inverse quand il affirme que l'émancipation des esclaves à Saint-Domingue, qui a mené au décret de février 1794, n'avait rien à voir avec le soulèvement des esclaves qui a commencé à Saint-Domingue en $1791^{36}$.

\section{Alan Forrest}

« Au cœur » est peut-être exagéré. Alors qu'il est clair que l'esclavage est apparu à certains comme un défi posé à l'idéologie révolutionnaire, il ne faut pas exagérer son importance pour les contemporains ou le degré de consensus qu'il a suscité. La plupart des nombreux pamphlets qui circulaient en France à la veille de la Révolution et durant les révoltes des esclaves exprimaient une position défensive, soucieuse de maintenir les possessions coloniales de la France, inquiète d'un affaiblissement du commerce français ou soucieuse de ne pas accorder un avantage commercial à la Grande Bretagne. La violence ouverte des soulèvements et le massacre des familles de planteurs ont fait plus scandale que la violence sous-jacente de l'économie esclavagiste elle-même. Et quand Napoléon a rétabli l'esclavage en Martinique et en Guadeloupe, cela n'a pas provoqué une vague d'indignation en France, quels que soient les dégâts que cela a pu faire par la suite à sa réputation. L'esclavage n'a été à aucun moment la question dominante de la Révolution. Pourtant cela a affecté la perception que les étrangers ont eue de la Révolution et le sens qu'ils donnaient à la Révolution, à l'époque et de nos jours encore. La question de l'esclavage permet de nuancer notre appréhension de la place de la Révolution française dans le monde et de replacer la France dans un contexte géographique plus

(35) Laurent DuboIs, Avengers of the New World, The Story of the Haitian Revolution, Cambridge, Mass., Harvard University Press, 2004 ; Id., A Colony of Citizens : Revolution \& Slave Emancipation in the French Caribbean, 1787-1804, Chapel Hill, University of North Carolina Press, 2004.

(36) Jeremy Popkin, You Are All Free : The Haitian Revolution and the Abolition of Slavery, Cambridge, Cambridge University Press, 2010. 
large. Mais on est là loin de l'idée que l'esclavage est une question centrale pour la Révolution dans la France métropolitaine.

\section{Matthias Middell}

Étant donné le nombre de noirs qui vivaient en France à la fin du $\mathrm{XVIII}^{\mathrm{e}}$ siècle $^{37}$, il est sûr que le lien des Français avec les colonies ne devait pas leur sauter aux yeux. Seules certaines parties de l'économie française étaient liées aux plantations et l'interrogation des planteurs sur les profits à tirer de l'esclavage a longtemps été maintenue à la marge de l'attention générale, mais pas définitivement. L'histoire de l'esclavage se laisse difficilement réduire à cette relation bilatérale entre la France et les Caraïbes. Nous en savons encore trop peu sur les conséquences globales des freins imposés à la traite et de l'interdiction officielle de l'esclavage. Et nous savons particulièrement peu de chose sur les participants et les termes du débat. Il serait intéressant de savoir dans quelle mesure les contemporains ont été conscients de la dimension globale du complexe plantation-esclavage.

\section{Karine Rance}

L'importance du fait militaire non seulement dans le processus révolutionnaire mais aussi dans les causes de la Révolution française (poids du coût de la guerre de Sept Ans et de la guerre d'Indépendance américaine) est connu. Lynn Hunt invite à revenir sur la rivalité franco-anglaise et sur le sens à donner à l'extension du conflit aux marges des empires coloniaux pour se demander si les colonies sont la cause du conflit ou si les guerres dans les colonies sont une conséquence du conflit européen.

\section{Alan Forrest}

Les guerres françaises du XVIII ${ }^{\mathrm{e}}$ siècle ont généralement été expliquées en termes d'ambitions diplomatiques et dynastiques en Europe, mais toutes avaient une dimension coloniale. L'Espagne, le Portugal, les Provinces-Unies étaient toutes des puissances coloniales importantes. Leur richesse dépendait profondément de leur empire. Ils considéraient leurs intérêts financiers et commerciaux comme déterminants pour leur richesse et pour leur poids en Europe. Et la Grande Bretagne, bien qu'étant tenue par des traités sur le continent et par la connexion hanovrienne, voyait ses colonies comme des possessions qui étaient aussi importantes pour la 
mère-patrie que n'importe quelle conquête en Europe. La guerre de Sept Ans commence dans les colonies et les guerres révolutionnaires, particulièrement quand elles sont vues de Londres, sont largement la continuation de la longue lutte franco-anglaise, autant pour les colonies que pour la maîtrise idéologique et territoriale du continent européen. Napoléon a sans doute considéré la base de sa puissance comme étant essentiellement européenne, mais il a gardé une attitude pour le moins ambivalente à l'égard des intérêts français en Amérique du Nord, comme la vente de la Louisiane le montre. Mais les autres puissances - l'Angleterre en tête - n'avaient aucune raison de suivre son exemple.

\section{Matthias Middell}

Les dynamiques à partir desquelles la Révolution s'est développée et celles que la Révolution a elle-même initiées sont d'une énorme complexité et ont une dimension globale. Est-ce que tout le monde, en France et ailleurs, a pris conscience de cette complexité immédiatement? Je ne le crois pas car cela a peu de sens de parler d'une «France » unifiée. Bien au contraire, les intérêts des différents groupes sociaux et ceux que les sensibilités régionales divergentes ont exprimés, ont déclenché un conflit politique durable. On a essayé, il y a quelques années, de présenter la Révolution comme une transformation culturelle et comme un immense processus de communication. Grâce à cela, nous en savons aujourd'hui beaucoup plus sur la recherche d'une classification du savoir disponible pour mener des stratégies et sur les nouvelles catégories permettant l'expression de ces stratégies et leur légitimation. On pourrait lire la question coloniale à travers le prisme de la concurrence avec l'Angleterre, mais on pourrait tout aussi bien attribuer à l'abolition (ou au contraire au maintien) de l'esclavage une priorité sur tout autre événement. La Révolution est apparue à beaucoup de participants comme une table rase après la disparition de l'Ancien Régime, justement parce qu'elle était en rupture avec les anciennes priorités - de même du côté de la Contre-Révolution, on ne se contentait pas de reprendre les anciennes solutions. Les Parlements, les sociétés populaires, les journaux et les revues, les théâtres et bien sûr la « rue » sont devenus le lieu d'une agitation qui s'est étendue car nombreux sont ceux qui partageaient le sentiment que plus rien n'était comme avant.

\section{Karine Rance}

Pour Christopher Bayly, la période révolutionnaire est la matrice des mondes modernes marquée par le passage d'une mondialisation archaïque fondée sur des échanges entre des empires aux limites imprécises à un 
système d'État-nations avec des sociétés de plus en plus uniformisées malgré des frontières mieux définies ${ }^{38}$. Adhérez-vous à cette vision globale de la période?

\section{Lynn Hunt}

Christopher A. Bayly considère la Révolution française comme un simple exemple de la crise mondiale entre les années 1720 et 1820 qui inclut les Safavides en Perse et la fragmentation de l'autorité moghole en Asie du Sud, mais il lui donne quand même une forte prééminence : la Révolution française a mobilisé de nouvelles énergies politiques et militaires et de ce creuset ont émergé des États plus forts, en France puis en conséquence parmi ses adversaires. Ces États européens renforcés, particulièrement l'État britannique, ont entrepris de déséquilibrer l'Asie avec une nouvelle combativitée $^{39}$. En d'autres termes, la spécificité de l'impérialisme européen du $\mathrm{XIX}^{\mathrm{e}}$ siècle trouve ses origines dans les guerres révolutionnaires.

\section{Paul Cheney}

Si une approche chronologique réduite peut apporter des perspectives, une chronologie plus large comme celle de Bayly peut aider les historiens à comprendre la signification globale à long terme de la Révolution. Jeremy Adelman a désigné la période d'indépendance en Amérique latine comme la fin d'une crise de souveraineté impériale qui a commencé au cours de la guerre de Sept Ans ${ }^{40}$. John H. Elliott voit une crise similaire dans l'Empire britannique, même s'il met en arrière-plan le problème du capitalisme, des élites marchandes et de la place centrale d'une économie de plantation. Mais le fil conducteur de ces récits est le montant croissant des coûts de protection dans les empires britannique et espagnol et le besoin de repenser les structures de la monarchie composite - la distribution des droits, le profit et l'autonomie qu'Adelman résume avec le terme « souveraineté », à une époque charnière du capitalisme mondial. Ceci fait écho aux travaux de Bayly, particulièrement avec l'idée que la globalisation archaïque a atteint son terme pendant cette période.

(38) Christopher A. BAYLY, The Birth of Modern World, 1780-1914 : Global Connections and Comparisons, Oxford, 2004 [trad. La naissance du monde moderne (1780-1914), Paris, les éditions de l'Atelier, 2007].

(39) Voir Christopher BAYLY, op.cit., particulièrement p. 96-100.

(40) Jeremy ADELMAN, Sovereignty and Revolution in the Iberian Atlantic, Princeton, Princeton University Press, 2006. 


\begin{abstract}
Alan Forrest
L'approche globale de Christopher Bayly et le cadre chronologique qu'il propose pour l'ère des Révolutions, soulève une question intéressante (et qui a été reprise dans d'autres domaines : on pense par exemple à l'ère des guerres révolutionnaires de Stig Førster). La Révolution française a d'abord combattu par la politique et par les idées ; elle utilisait le langage de l'individualisme et des droits politiques et cela est resté au cœur de sa dynamique, même si elle s'est aussi intéressée à la question du libéralisme économique. En adoptant l'idée de souveraineté nationale, elle a aidé à créer dans l'Europe du XIX ${ }^{\mathrm{e}}$ siècle des États-nations qui ont affaibli les empires multinationaux du vieux continent, en Autriche et en Turquie. En retour cela a conduit à un nouveau type d'empire extra-européen, de dimension mondiale, au XIX ${ }^{\mathrm{e}}$ siècle, à commencer par la Grande Bretagne et la France elle-même bien sûr. Le risque est d'attribuer trop de choses à la Révolution française. Il paraît bien plus facile de lier les événements français à un monde atlantique plus large quand on cherche à définir les causes de 1789 ou à évoquer l'impact de la Révolution dans le monde post-révolutionnaire, que d'expliquer dans cette optique l'évolution spécifique de la Révolution en France. Il est tout à fait défendable d' affirmer que dans les choix faits (les attaques faites envers la religion, l'insistance sur la poursuite du centralisme jacobin, le rejet du modèle anglo-saxon de la séparation des pouvoirs) la Révolution plonge ses racines dans la société française et la monarchie des Bourbon. L'histoire globale ajoute une dimension importante à l'histoire de la Révolution française mais elle ne peut pas totalement supplanter les autres approches.
\end{abstract}

\title{
Karine Rance
}

L'un des problèmes de la perspective globale est la périodisation. L' « ère des révolutions », avec l'idée de plusieurs foyers révolutionnaires concomitants, connectés les uns aux autres, débute pour David Armitage avec la Guerre de Sept Ans (1756-63) et se termine à la guerre sinobritannique de l'Opium (1839-42). Est-ce qu'un tel découpage spatiotemporel n'aboutit pas à une catégorie « révolution » très flexible et à une périodisation qui ne se justifie par forcément en Europe occidentale puisque la Révolution de 1848 s'en trouve exclue ?

\section{Alan Forrest}

Je suppose que les dates pour une ère des révolutions vont toujours être subjectives. David Armitage insiste sur la nécessité de la faire 


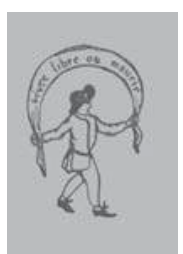

commencer avec la guerre de Sept Ans, puisque c'était une guerre qui a commencé dans les colonies et où les batailles dans les Amériques ont eu autant d'importance que le conflit en Europe. Du point de vue du conflit franco-anglais, il y eut une continuité claire entre d'une part 1756 et la guerre d'indépendance américaine, et d'autre part les guerres de la Révolution et de l'Empire. Mais il faut être conscient que c'est encore une manière très euro-centrique de regarder le monde. La date finale est beaucoup plus ouverte au débat car différents événements peuvent sembler également valables selon le point de vue. L'esclavage ne peut pas vraiment être déterminant, puisque pour les États-Unis cela irait jusqu'en 1865 et pour l'Amérique du Sud jusqu'en 1884, ce qui rend la période si élastique qu'elle perd presque tout sens. Les guerres de l'Opium ont le mérite de déplacer le conflit vers un autre hémisphère et vers le clash entre la Chine et l'empire britannique en Orient. Mais, sauf si on cherche volontairement à exclure l'Europe de ce contexte global, je ne vois pas de raison de s'arrêter avant 1848 : une date clé pour l'Europe, bien sûr, mais aussi le moment de la seconde abolition de l'esclavage dans les colonies françaises - mais pas la fin de l'esclavage atlantique, nous sommes d'accord. Je crains qu'il n'y ait pas de réponse qui puisse faire autorité car une périodisation de ce type ne peut être qu'une question de préférence individuelle.

\section{Matthias Middell}

Pour ce qui est de la périodisation, plusieurs critères s'entremêlent dans les discussions récentes sur l'histoire globale des $\mathrm{XVIII}^{\mathrm{e}}, \mathrm{XIX}^{\mathrm{e}}$ et $\mathrm{XX}^{\mathrm{e}}$ siècles. Si on considère la Révolution française comme faisant partie d'un processus réussi de passage à une nouvelle organisation des sociétés en réaction aux dynamiques de ce qu'on appelle la globalisation archaïque (c'est-à-dire une globalisation qui a encore devant soi l'énorme accélération des communications et des transports qui a lieu à partir du milieu du XIX ${ }^{\mathrm{e}}$ siècle), alors le début de cette crise remonte certainement simplement à la guerre de Sept Ans. Mais la mondialisation des conflits et des discussions sur la possibilité d'une modernisation des empires prend une nouvelle couleur après 1756. Michael Geyer et Charles Bright ont montré, dans un article ayant reçu un large écho, que les révolutions, les guerres et les guerres civiles entre 1840 et 1880 reconstruisent un complexe, qui montre l'intensité de la recherche d'une solution à la crise caractérisée par l'émergence de l'industrie et du marché mondial des capitaux et des produits. On peut ainsi distinguer la période qui va de 1700 à 1760 environ (avec des réponses encore assez isolées à la crise provoquée de l'Inde à l'Europe par la globalisation archaïque) de la période qui va de 1760 aux 
années 1830 (marquée par une interdépendance croissante de la recherche des solutions à la crise pour lesquelles les empires, avec leur système de privilèges multiples, ne paraissent plus adaptés). À cela s'ajoute la période 1840-1880 comme rupture avec ce qu'on appelle la condition globale, dans laquelle le modèle de la globalisation moderne est de plus en plus visible. De cette manière on peut essayer de relier les résultats de l'histoire économique et financière avec l'histoire des relations internationales et l'histoire sociale qui se concentre sur les transformations (révolutionnaires et réformatrices) des sociétés. Ceci n'est toutefois qu'une hypothèse, car il reste encore beaucoup de régions du monde sur lesquelles on en sait trop peu.

\section{Karine Rance}

Quelles perspectives voyez-vous se dessiner?

\section{Lynn Hunt}

Cheney approche la globalisation par une étude de la pensée économique, ce qui est certainement un bon point de départ. Ce qu'il faut maintenant c'est une analyse à la fois théorique et empirique de la globalisation du XVIII ${ }^{\mathrm{e}}$ siècle et de son impact sur la Révolution française. Dans un volume récent ${ }^{41}$, un groupe d'universitaires (surtout Américains mais comprenant aussi Pierre Serna et l'Australien Ian Coller) ont essayé de pousser la perspective globale dans de nouvelles directions, en se concentrant sur les causes et conséquences politiques et économiques plus que sur la pensée économique. Michael Kwass par exemple montre que l'opposition politique à la monarchie émerge d'une part en réaction à la dure répression de la contrebande de calicots de coton indien interdits et du tabac contrôlé par le gouvernement. Dans le même livre, je montre dans mon article sur les origines financières globales de 1789 que l'effondrement financier de 1789 ne peut pas être compris sans prendre en compte dans l'ensemble des facteurs l'essor de fortunes issues du commerce des esclaves et du commerce de l'Océan Indien dans les années 1780. La monarchie n'était pas affaiblie par le montant de ses dettes ou même par la prime de risque qu'elle était obligée de payer pour emprunter ; la forte croissance commerciale après la paix de 1783 a encouragé la monarchie à continuer à emprunter jusqu'à ce qu'elle se trouve au bord du gouffre et qu'elle y fasse une chute, laquelle avait été préparée par les banquiers qui avaient afflué à Paris pour y 


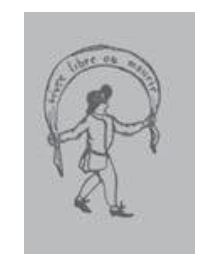

gagner de l'argent sur les emprunts souverains. D'autres articles du même ouvrage attirent l'attention sur les sources coloniales, transeuropéennes et atlantique de l'universalisme et du cosmopolitisme révolutionnaire, et sur l'influence d'événements ayant eu lieu ailleurs comme en Égypte, sur le développement des politiques et pratiques françaises.

Mettre la Révolution française dans le contexte de la globalisation du commerce, des idées et des pratiques peut aider à expliquer ses origines et sa dimension globale, ce qui est un objectif louable en soi. Cela pourrait aussi être l'occasion de mettre la Révolution française sur la table pour une discussion sur les approches globales, impériale et atlantique de l'histoire. Mais comme David Bell l'a affirmé récemment («Questioning the Global Turn : The Case of the French Revolution »), ces nouvelles approches ne parviennent pas à expliquer certains développements importants de la Révolution : notamment les conflits sur la réorganisation de l'Église catholique, l'intensité de la radicalisation, et la Terreur. La perspective atlantiste de Palmer et Godechot a attiré l'attention sur la commune volonté de renverser l'aristocratie et d'établir des formes de participation démocratique - aspirations trop souvent absentes des analyses globales récentes - mais l'une des critiques majeures qui leur a été adressée tient toujours : malgré tous les points communs entre les révolutions belges, néerlandaise et française par exemple, personne ne considère les soulèvements belge ou néerlandais comme paradigmatiques de la politique moderne. La Révolution française occupe encore une place particulière et les comparaisons entre les empires britannique, espagnol, safavide ou mongol qui n'insistent que sur les points communs vont inévitablement faire oublier ce qui rend la Révolution française encore intéressante et utile aujourd'hui : l'ambition de créer quelque chose de radicalement neuf dans l'histoire.

\section{Paul Cheney}

Si les historiens veulent rester attentifs aux sources et aux formes du pouvoir, ils doivent continuer à penser les histoires supra-nationales (qu'elles soient globales, impériales ou océaniques), en termes de réseaux constitutifs : même quand les historiens sont intéressés par des phénomènes culturels connectés qu'ils voudraient placer dans un contexte largement nonéconomique, ils doivent souvent cheminer le long des routes commerciales des empires historiques. Le travail de Subrahmanyam sur les processus de métissage culturel et de circulation des élites dans l'empire portugais le montre très clairement. L'histoire supra-nationale de la Révolution française devrait être connectée, à condition de ne pas oublier que la 
plupart des connexions dont il est question étaient d'abord issues de l'empire commercial français, dont la partie la plus lucrative (et la plus sujette aux conflits) était située dans le monde atlantique.

\section{Alan Forrest}

Mettre la Révolution française dans un cadre atlantique et colonial et en rapport avec des dynamiques issues d'autres parties du globe enrichit notre compréhension des circonstances dont elle a émergé et des choix auxquels les révolutionnaires ont été confrontés. Cela joue aussi un rôle majeur dans l'explication des causes et des buts de guerre de cette période. La guerre en arrive à dominer les priorités de la Révolution. Cela a déplacé des millions de soldats à travers le continent et au-delà, les mettant en contact avec de larges parties du monde et les exposant à des expériences qui les ont marqués pour le reste de leur vie. Cela a aussi déplacé des populations civiles, détruit des moyens d'existence et poussé des communautés entières à une migration forcée. Certains n'ont jamais pu accepter un retour dans le monde qu'ils avaient laissé derrière, comme ces soldats de Napoléon qui se sont jetés au service des mouvements anti-colonialistes du Mexique, au Pérou et au Chili à partir des années 1810. La guerre, autant que la Révolution, a été une expérience globalisante.

\section{Matthias Middell}

Depuis la fin du XVIII ${ }^{\mathrm{e}}$ siècle, chaque époque s'est intéressée à la Révolution française sous un angle spécifique. Dans un premier temps, la Révolution semblait bien loin de la question de l'adaptation des sociétés à la globalisation. Ceci a changé récemment. L'explication de la Révolution met en rapport plusieurs facteurs dont les origines sont manifestement extra-hexagonales. On ne se représente plus la société comme un container dans lequel les transformations sociales ont des origines exclusivement — ou en tout cas essentiellement - internes. Ce principe fondamental de l'histoire globale nous oblige à répondre de manière beaucoup plus précise à la question de savoir ce qu'on entend par globalisation (archaïque ou moderne). Mais il n'y a pour l'instant que des réponses partielles.

L'histoire globale s'intéresse beaucoup à l'espace comme référence centrale de l'organisation des communautés sociales. C'est d'autant plus difficile à étudier que les références spatiales s'entrecroisent et que les acteurs font preuve d'une aptitude plus ou moins grande au jeu d'échelle. De mon point de vue, la question centrale de l'histoire globale pour les prochaines années est de savoir comment, en réponse aux différents « espaces » que les individus et les groupes considéraient comme significatifs pour 
eux, un régime de territorialisation a été conçu, qui a paru adapté à une certaine période pour contrôler les anciens et nouveaux courants de capitaux, d'individus, de biens et d'idées. La Révolution française ouvre pour cela un fantastique champ d'expérimentation car les débats y ont été aussi explicites que polémiques. Observer la déconstruction des «espaces » significatifs pour la France comme réponse à une globalisation qui a mené à la banqueroute de l'État offre la possibilité de jeter une nouvelle lumière sur une histoire apparemment connue et suffisamment étudiée.

Traduit de l'allemand et de l'anglais par Karine Rance

Paul CHENEY

University of Chicago

cheney@uchicago.edu

Alan FORREST

University of York

alan.forrest@york.ac.uk

Lynn HuNT

UCLA

lhunt@history.ucla.edu

Matthias MidDELL

Universität Leipzig

middell@rz.uni-leipzig.de

Karine RANCE

Université Blaise Pascal, Clermont-Ferrand

karine.rance@univ-bpclermont.fr 